\title{
Hybrid Fourier Domain Modelocked Laser utilizing a Fiber Optical Parametric Amplifier and an Erbium Doped Fiber Amplifier
}

\author{
Kyle H. Y. Cheng ${ }^{\mathrm{a}}$, Beau A. Standish ${ }^{\mathrm{b}}$, Victor X. D. Yang ${ }^{\text {b,c,de }}$, K. K. Y. Cheung, \\ Xijia Gu ${ }^{b}$, Edmund Y. Lam ${ }^{\mathrm{a}}$, K. K. Y. Wong ${ }^{{ }^{\mathrm{a}}}$ \\ ${ }^{a}$ Department of Electrical and Electronic Engineering, the University of Hong Kong, Hong Kong, \\ China PRC \\ ${ }^{\mathrm{b}}$ Department Electrical and Computer Engineering, Ryerson University, Toronto, Canada \\ ${ }^{c}$ Department of Physics, Ryerson University, Toronto, Canada \\ ${ }^{\mathrm{d}}$ Division of Neurosurgery, University of Toronto, Toronto, Canada \\ ${ }^{\mathrm{e}}$ Imaging Research, Sunnybrook Health Sciences Center, Toronto, Canada
}

\begin{abstract}
To our knowledge, we report the first Fourier domain modelocked laser (FDML) constructed using optical parameter amplifier (OPA) in conjunction with an erbium-doped fiber amplifier (EDFA), centered at $\sim 1556 \mathrm{~nm}$. We utilized a onepump OPA and a C-band EDFA in a series configuration with a polygon-grating wavelength filter to generate a hybrid FDML spectrum. Results demonstrate a substantially higher output power, better spectral shape and significantly more stable bandwidth than individual configurations. We believe this technique has the potential to enable several amplifiers to complement individual deficiencies resulting in improved spectral shapes and power generation for imaging applications such as optical coherence tomography (OCT).
\end{abstract}

Keywords: Fourier domain modelocking, laser, optical parametric amplifier, swept-source

\section{INTRODUCTION}

Recently, swept source (SS) lasers have become a standard laser configuration for sensing applications [1-3], with particular interest in the field of biomedical imaging. Optical Coherence Tomography (OCT) is an emerging imaging technique that generates cross-sectional images of biological tissue in-vivo with axial resolutions approaching histological standards $(\sim 1-10 \mu \mathrm{m})$. Several types of OCT platforms have been developed for the purpose of improving the signal-to-noise ratio, axial and transverse resolution and increasing the A-scan rate to acquire faster and larger threedimensional image sets. Of particular interest is the technique of SS-OCT, also called optical frequency domain imaging (OFDI), which is an imaging technique that is based on spectral interferometry [4]. A broadband laser source is optically filtered such that the wavelength that interacts with the sample is swept linearly in time. Recently, a new SS technique has been introduced to the field, namely, Fourier domain modelocking (FDML) developed by Huber et al [5]. In theory, it matches the fiber laser ring cavity length with the round trip time of the photons circulating in the cavity such that when one wavelength arrives at the filter, it combines with the same particular wavelength that is being generated. Therefore, different wavelengths can be continuously fed through the gain medium without substantial loss due to the rapidly tuning wavelength filter. Common filters used to sweep the optical wavelengths include piezo-electric driven tunable Fabry-Perot interferometer (TFPI) [5] and polygon-grating filters [6]. Compared with traditional SS, FDML can generate equivalent optical output power at a much lower pump power.

Semiconductor optical amplifiers (SOA) have been commonly used to construct FDML SS lasers due to the SOA's compact size and relatively wide bandwidth. However, there are several disadvantages of SOAs when compared with other kinds of optical amplifiers; for example, it has higher noise figure and lower gain compared with erbium-doped fiber amplifier (EDFA). Therefore it is sometimes more desirable to use optical amplifiers other than SOA. Previous studies have demonstrated the use of a SOA in FDML configuration in the $1050 \mathrm{~nm}$ regime [7] and the possibility of using Raman amplifier as gain medium in the $1550 \mathrm{~nm}$ regime [8]. However, the results had limited bandwidth and power. Another study demonstrated two SOAs in a parallel configuration [9], where the bandwidth was increased,

Fiber Lasers VII: Technology, Systems, and Applications, edited by Kanishka Tankala, Jay W. Dawson, Proc. of SPIE Vol. $7580,75802 Q \cdot$ C 2010 SPIE · CCC code: 0277-786X/10/\$18 - doi: 10.1117/12.842720 
however it involved the coupling of the two paths yielding power loss. An additional disadvantage with this setup was that the two paths needed to be of exact equal lengths or the FDML operation would be compromised.

A plausible next step would include aligning two optical amplifiers with distinctive optical characteristics such that their combined advantage would be greater than their individual performance. In this study, two distinctive optical amplifiers, specifically an optical parametric amplifier (OPA) and an EDFA were utilized to construct a hybrid FDML laser. Results demonstrate that their combined performance out-performs their individual configurations resulting in a laser with better spectral bandwidth and shape.

\section{PRINCIPLE AND THEORY}

Most studies involving FDML utilize common optical amplifier gain mediums such as the SOA. As a standalone optical amplifier, it is energy efficient and operates with a relatively broad bandwidth of $\sim 100 \mathrm{~nm}$. Other competing optical amplifiers such as EDFA and Raman amplifier have a much narrower bandwidth and individually they may not be utilized for FDML configuration.

This is the first study to demonstrate a hybrid FDML configuration, which utilizes two distinctly different types of optical amplifiers, as aforementioned, a one-pump OPA and a C-band EDFA, to the best of our knowledge. Fiber OPAs are based on third-order non-linear susceptibility of glass fibers [10]. If one (or two, which depends on the actual configuration) strong pump(s) and a weak signal are fed into a fiber, an idler is generated. Signal and idler can grow together if pump power is high enough, and phase matching condition is satisfied. In other words, when an optical signal at angular frequency $\omega$, co-propagates in a fiber with a strong pump at $\omega_{\text {pump }}$, the signal is amplified and another frequency, called the idler is generated at $\omega_{\text {idler }}=2 \omega_{\text {pump }}-\omega_{\text {signal }}$. The idler contains the same modulation information as the input signal, with an inverted spectrum. The typical gain spectrum of an one-pump OPA shows a dip around the pump wavelength, while the C-band EDFA has an unevenly shaped gain spectrum from $1525 \mathrm{~nm}-1565 \mathrm{~nm}$. Therefore it would be advantageous to complement the dip by applying the hybrid FDML technique in the cavity.

The cavity is constructed similarly to a traditional ring cavity laser, but with the addition of a spool of $\sim 6 \mathrm{~km}$ single-mode fiber (SMF) for FDML operation and a polygon-grating filter or a piezo-electric driven TFPI [6].

\section{EXPERIMENTAL SETUP}

The schematic diagrams of the Hybrid FDML configuration implemented with polygon-grating filter and TFPI are shown in Figure 1a and b, respectively. The pump of the OPA is at the top of Figure 1. First, the pump optical wavelength was generated at $1556 \mathrm{~nm}$ by the tunable laser source. The light then passed through a phase modulator which was itself modulated by a pseudo-random binary sequence (PRBS) generator for stimulated Brillourin scattering (SBS) suppression [11]. The non-linear optical phenomenon, SBS, primarily limited the power level that could be launched into the highly non-linear fiber (HNLF). Afterwards, the pump went through two stages of EDFA amplification and then reached the HNLF for parametric amplification. A fiber Bragg grating (FBG) was used for attenuating the pump power in the cavity. The EDFA was placed after the TFPI for the hybrid FDML generation. The $\sim 6 \mathrm{~km}$ SMF fiber extended the cavity such that the round trip time of the light in the cavity was comparable to one period of the filter driving signal. The FDML effect was then generated by matching the sweeping period of the polygon-grating filter or the TFPI with one round-trip time of the light in the cavity. 


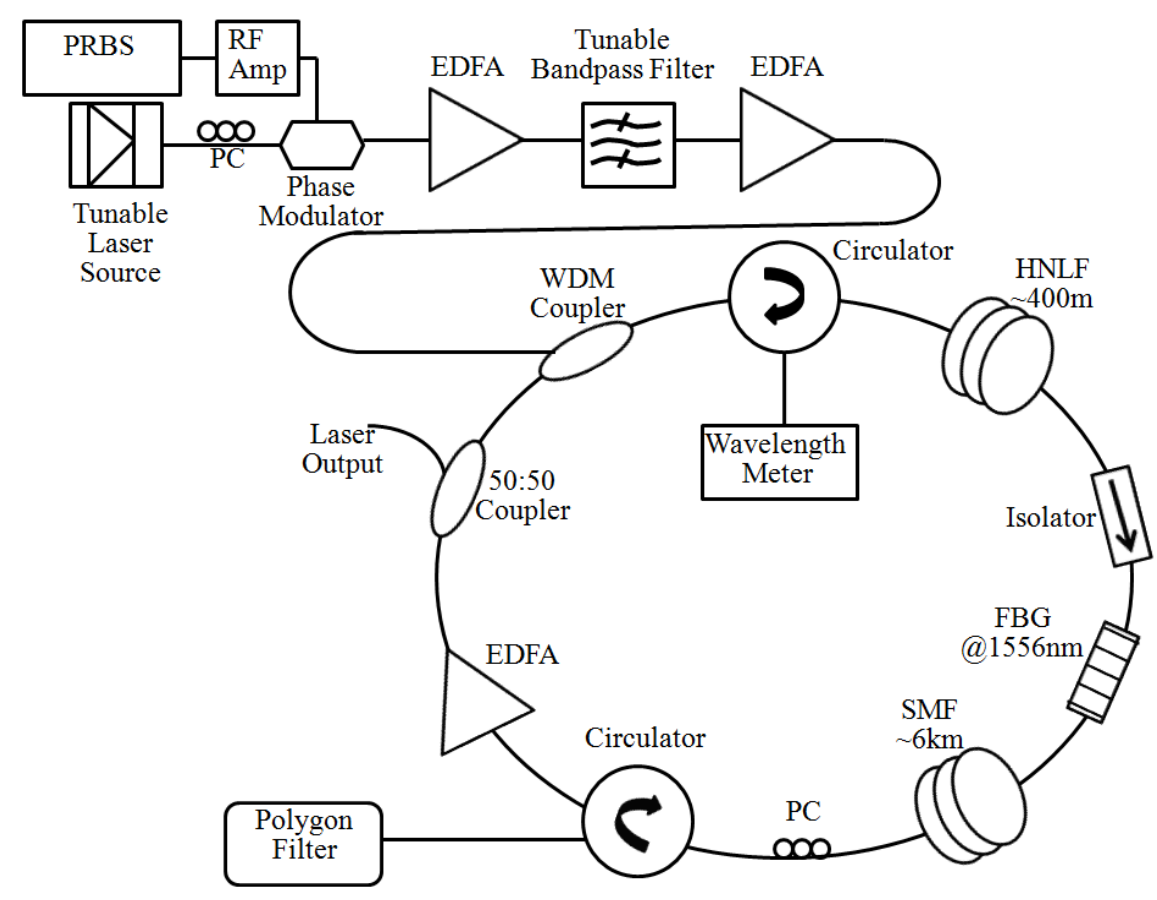

(a)

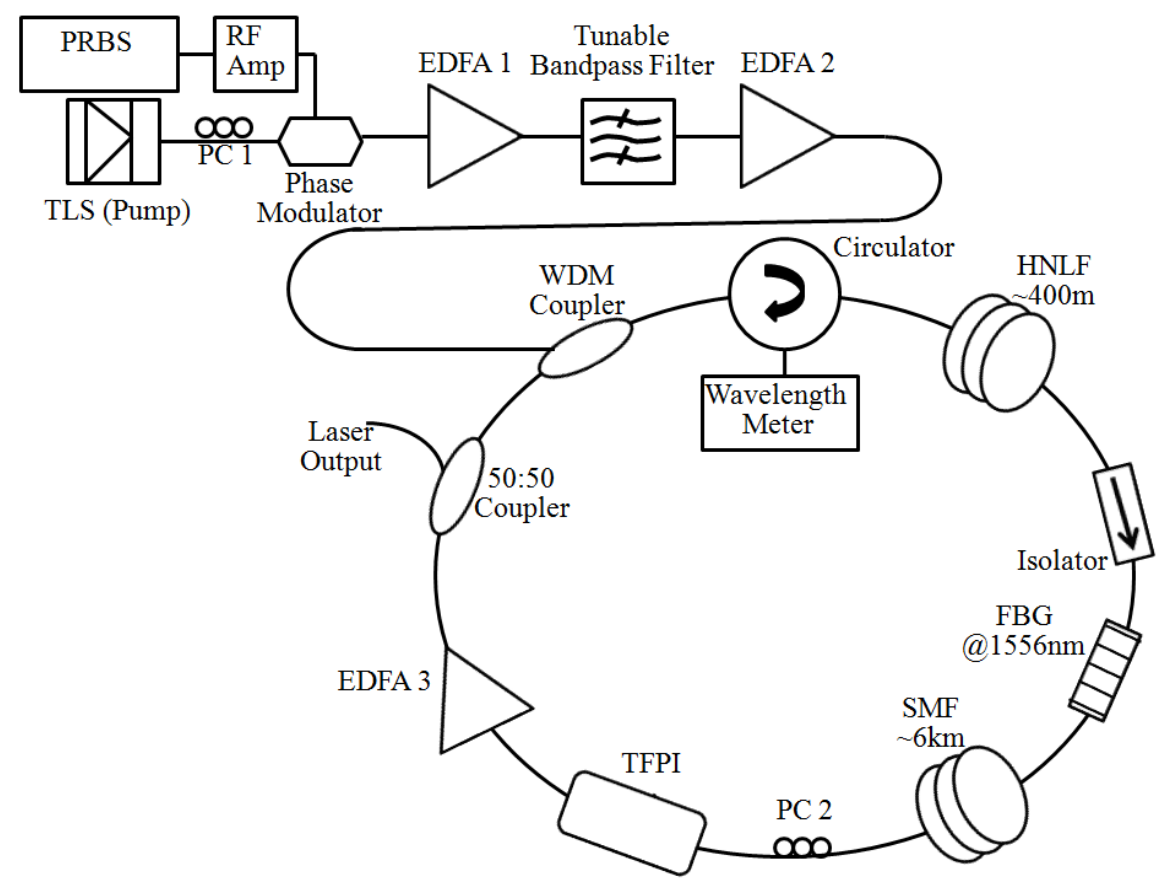

(b)

Figure 1. Schematic of the Hybrid FDML implemented with (a) polygon filter; (b) TFPI. EDFA: Erbium Doped Fiber Amplifier, FBG: fiber Bragg grating, HNLF: Highly Non-Linear Fiber, PC: Polarization Controller, PRBS: Psuedorandom Binary Sequence Generator, RF Amp: Radiofrequency Amplifier, SMF: Single Mode Fiber, WDM Coupler: Wavelength Division Multiplexer 


\section{RESULT AND DISCUSSION}

FDML spectra generated using the individual one-pump OPA and the C-band EDFA are demonstrated in Figure 2a and b, respectively.

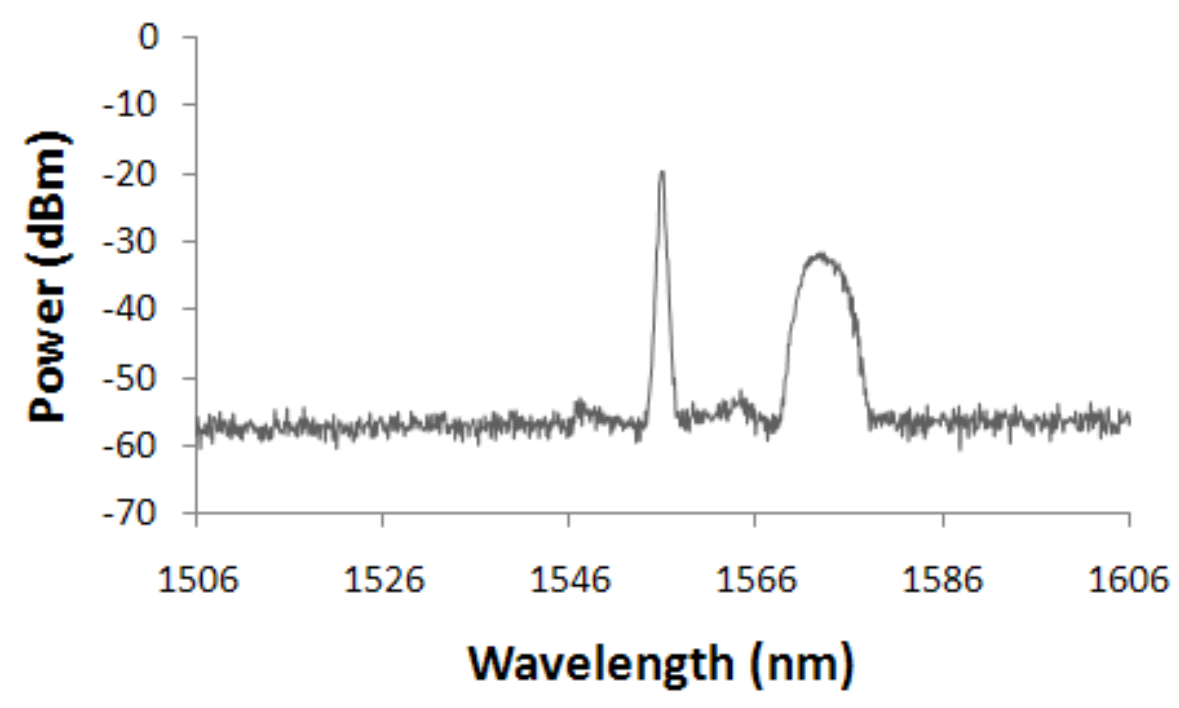

(a)

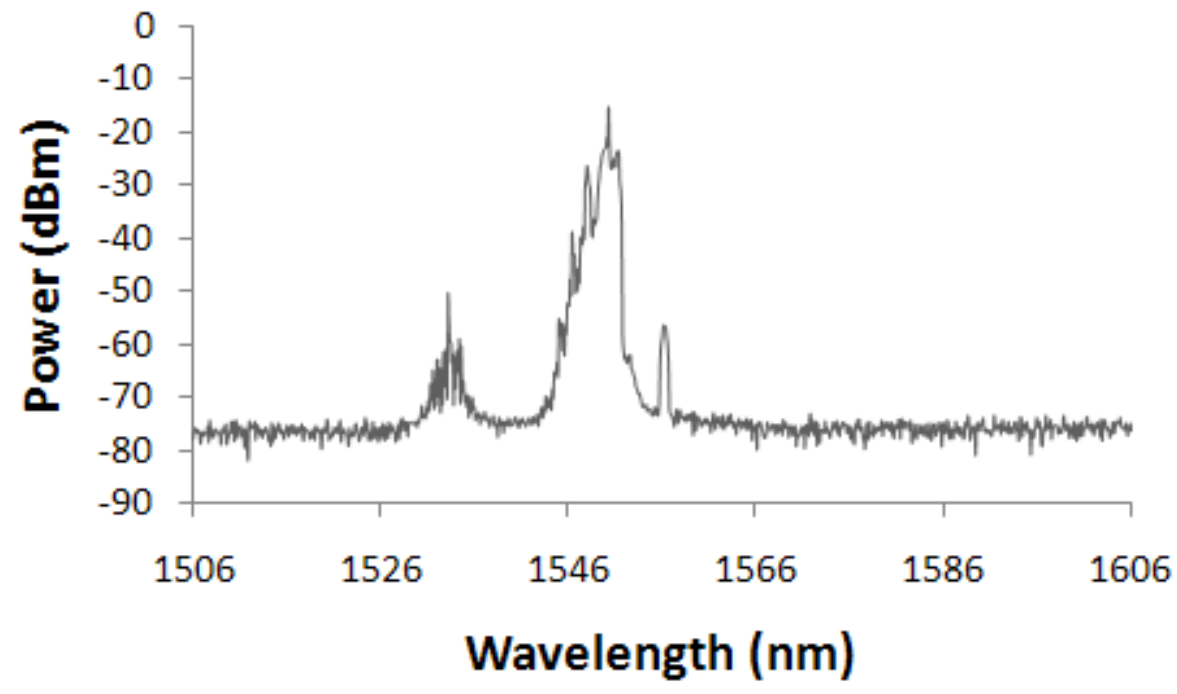

(b)

Figure 2. (a) FDML spectrum using OPA alone (only one side of the gain spectrum was utilized to create FDML) (Resolution: $0.5 \mathrm{~nm}$ ), the spike at $1556 \mathrm{~nm}$ is the pump; (b) FDML spectrum using only the C-band EDFA (Resolution: $0.5 \mathrm{~nm}$ )

From Figure 2a and $b$, it can be observed that the power and bandwidth of both OPA and EDFA in FDML configuration are not sufficient for imaging applications. In Figure 2a, when only one side of the OPA gain spectrum was utilized, the bandwidth was $\sim 10 \mathrm{~nm}$. In Figure $2 \mathrm{~b}$, when only EDFA was utilized, the uneven gain spectrum of the EDFA only generates $\sim 10 \mathrm{~nm}$ of usable FDML spectrum. Therefore, we proposed to combine these two amplifiers in the same cavity to generate a hybrid FDML effect with broadened overall operating bandwidth and better spectral shape.

Figure 3a shows the FDML spectrum obtained when both the one-pump OPA and the C-band EDFA were utilized to generate the hybrid FDML effect. The pump power of the OPA was $<2 \mathrm{~W}$. However, the setting depends on many factors 
such as the lengths of the HNLF and the erbium-doped fiber in the EDFA and should not be taken as an absolute requirement. In FDML operation the longer-wavelength side of the OPA gain spectrum compensated the spectral unevenness in of the EDFA, while the dip in the M-shaped one-pump OPA gain spectrum was reduced and partially filled by the EDFA gain spectrum. The resulting output power was also improved significantly as can be compared between Figure 2 and Figure 3a. Nearly every wavelength in the vicinity of the swept range experienced more than 10dB increase in output power.

The spectrum in Figure $3 b$ was obtained when the polygon-grating filter driving frequency was detuned by $\sim 300 \mathrm{~Hz}$. When the filter was not driven with the resonant frequency, the output power in the swept range decreased substantially. Figure $3 b$ therefore proves that Figure $3 \mathrm{a}$ is indeed in FDML operation.

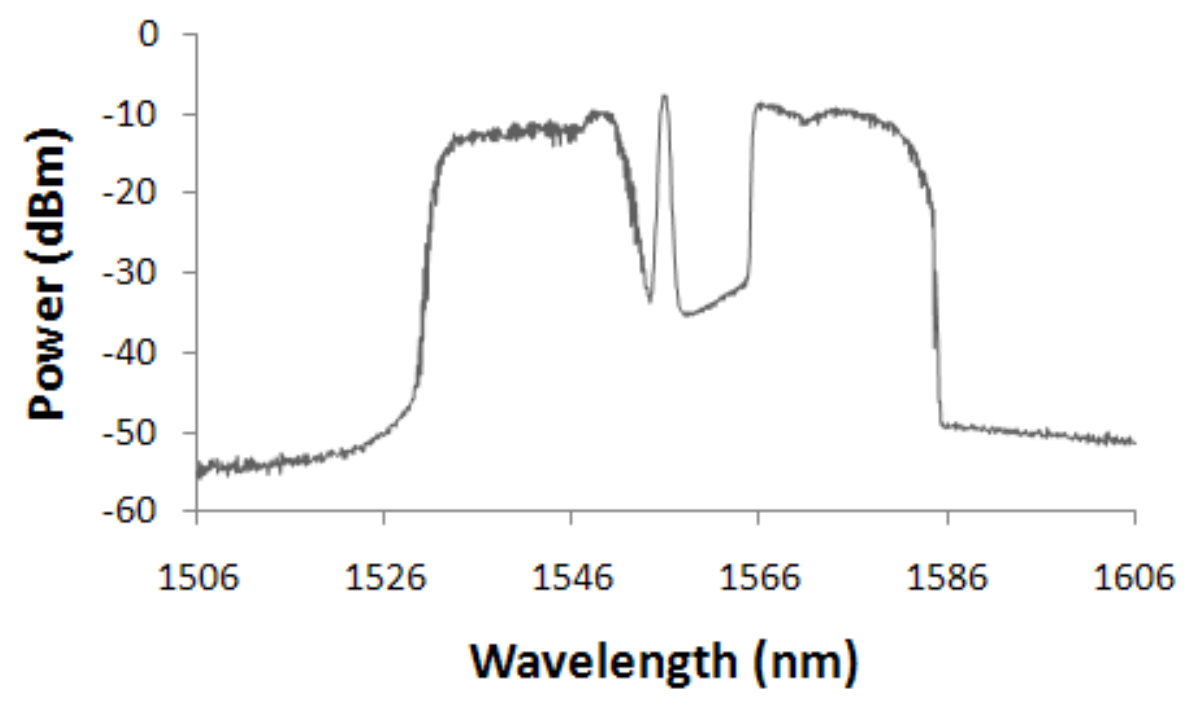

(a)

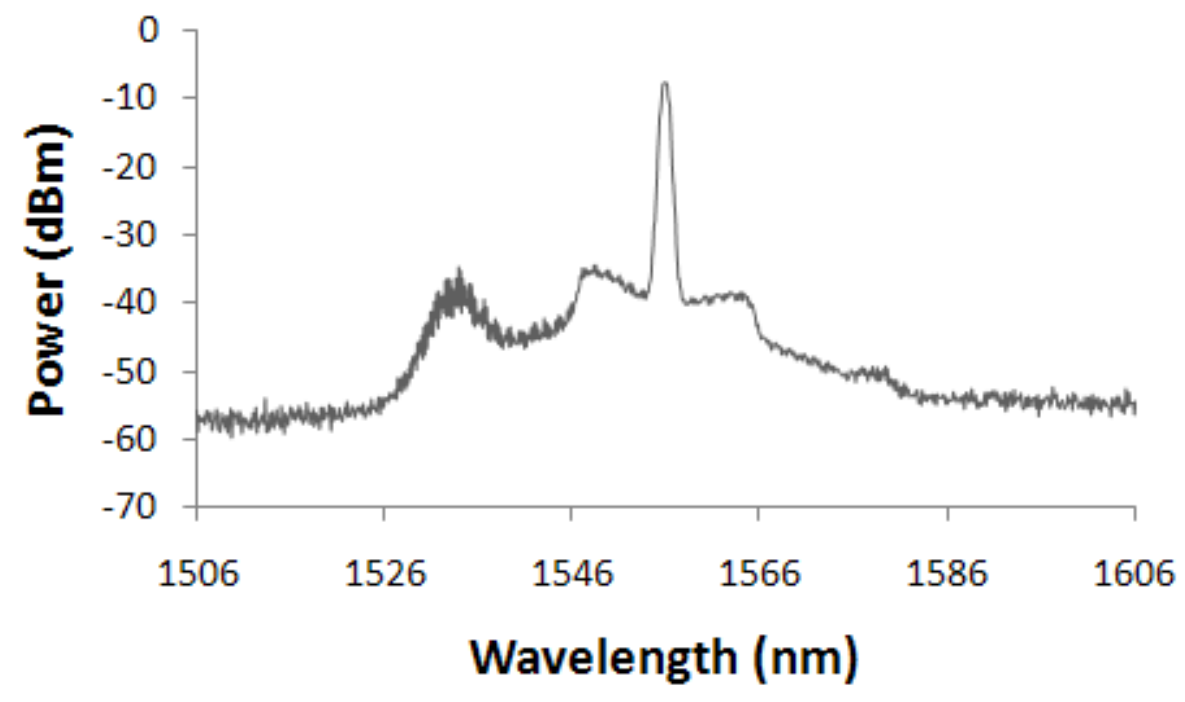

(b)

Figure 3. (a) Hybrid FDML spectrum (the central notch may be due to the power loss from the WDM Coupler) (Resolution: $0.5 \mathrm{~nm}$ ); (b) Spectrum when the polygon driving frequency was detuned from FDML operation

(Resolution: $0.5 \mathrm{~nm}$ ) 
Figure 4a and $\mathrm{b}$ show the hybrid FDML spectrum implemented with the TFPI. Result shows that with TFPI, the spectrum generated contains more amplified spontaneous emission (ASE) and swept less bandwidth than the hybrid FDML implemented with the polygon-grating filter. Therefore, it may be more desirable to use polygon filters to implement such kind of setup.

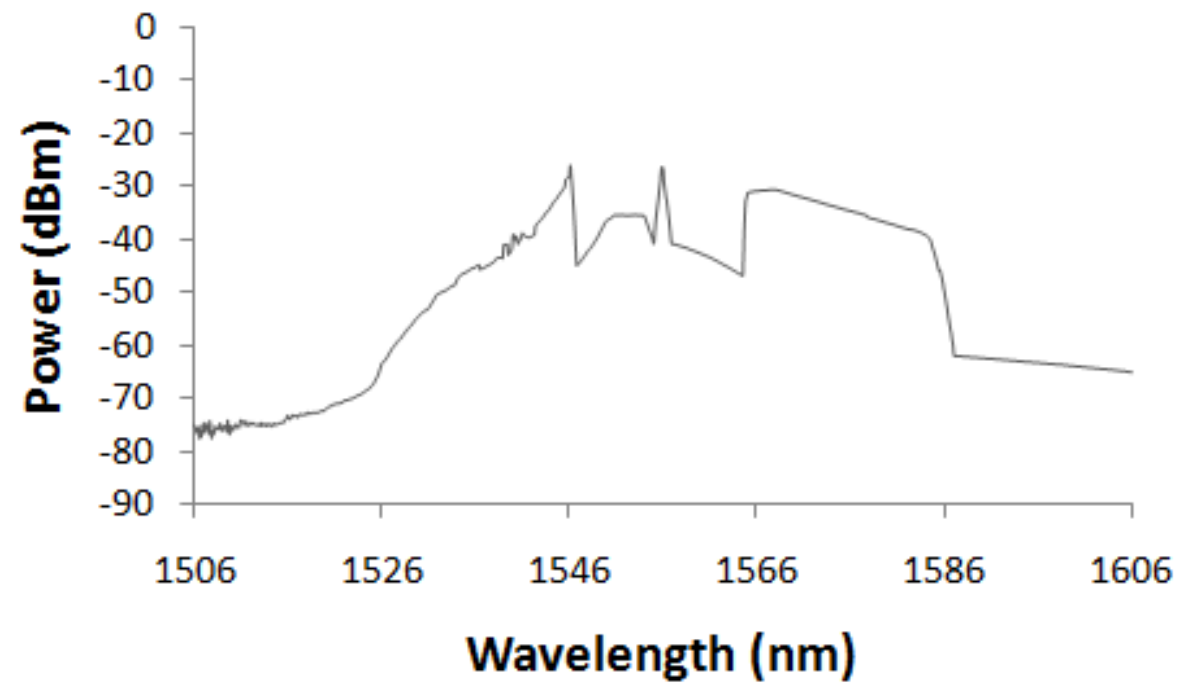

Figure 4. Hybrid FDML spectrum using TFPI (Resolution: 0.5nm)

The previous parallel SOA configuration was designed to allow broader overall bandwidth [9]. Essentially the left side of the spectrum was dominated by one SOA and the right side by another. In our design, the series configuration showed similar performance and was much easier to setup FDML in a series configuration. The design of this new cavity included the placement of the EDFA after the polygon filter to generate the hybrid FDML. This was crucial because if the EDFA were put before the polygon filter and immediately after the OPA (see Fig. 1), the resulting spectrum would be much more discontinuous and exhibit lower output power. Interestingly, the left side of the spectrum would be dominated by EDFA gain and the right side would be dominated by OPA gain. It was observed that different wavelength regions of the resulting spectrum would conform to the shapes of the corresponding regions of the gain spectra of individual optical amplifiers with higher gain. In this case, on the left side of the pump, the EDFA gain was much stronger than OPA gain, resulting in the left side to be dominated by EDFA gain. Conversely, on the right side of the spectrum the OPA gain was much stronger, therefore the OPA gain dominated in the right part of the spectrum. This observation of spectral distribution based on amplifier power requires investigation and continues to be an active area of research within our group. Moreover, if the two SOAs in [9] were placed in series operation in the cavity, there may be absorption issues since part of their gain spectra overlaps. This would mean that some of electron energy levels would be the same resulting in absorption of photons in the latter SOA. In our cavity design of the cavity, this may be alleviated as the filter sweeps through individual wavelengths and therefore reduces the influence of one amplifier on the other, therefore allowing more output power.

Furthermore, a one-pump OPA was pursued in this proof-of-principle study. However, two-pump OPAs are readily available that could be used to generate a continuous flattened spectrum with wider bandwidth [10] or even polarization independent operation [12]. The versatile gain spectrum generation at arbitrary wavelength regions of OPA may enable its incorporation into any kind of hybrid FDML configuration with other complimentary type of optical amplifiers (e.g. SOA or Raman amplifiers), which further enhances the bandwidth and/or power of existing FDML laser systems.

\section{CONCLUSION}

In conclusion, we have demonstrated a hybrid FDML laser that utilized distinct optical amplifiers to generate a "crossreinforcing" FDML spectrum. In this operation we observed significant improvements in the lasing characteristics including wider spectral bandwidth and higher output power when compared to the individual operation of the amplifiers. We believe that this configuration is not limited to only OPA or EDFA. Other optical amplifiers are currently 
being investigated (e.g. SOA or Raman amplifier) to complement individual deficiencies. Lasers with extended useable bandwidth and maximized output power are expected to be created from the hybrid FDML design for imaging use in modalities such as OCT.

We have also demonstrated that it is possible to operate in a FDML configuration through the implementation of an OPA. With the versatility of the center wavelength selection of an OPA and the wide bandwidth (possibly $>400 \mathrm{~nm}$ in other demonstrations [13]), the use of an OPA in combination with additional optical amplifiers in a hybrid FDML configuration could be utilized to provide ultrahigh resolution OCT imaging.

\section{ACKNOWLEDGMENT}

This work was supported in part by the Research Grants Council of the Hong Kong Special Administrative Region, China under Projects HKU 7139/06E, HKU 7172/07E and HKU 7179/08E, the Canadian Foundation for Innovation, and the Canada Research Chair program. The authors would also like to acknowledge Sumitomo Electric Industries for providing the HNLF.

\section{REFERENCES}

[1] Jung, E. J., Kim, C.-S., Jeong, M. Y., Kim, M. K., Jeon, M. Y., Jung, W. and Chen, Z., "Characterization of FBG sensor interrogation based on a FDML wavelength swept laser," Opt. Express 16, 16552-16560 (2008).

[2] Chen, D., Shu, C. and He, S. , "Multiple fiber Bragg grating interrogation based on a spectrum-limited Fourier domain mode-locking fiber laser," Opt. Lett. 33, 1395-1397 (2008).

[3] Kranendonk, L. A., Walewski, J. W., Sanders, S. T., Huber, R. J. and Fujimoto, J. G. , "Measurements of Gas Temperature in an HCCI Engine by Use of a Fourier-Domain Mode-Locking Laser," Laser Applications to Chemical, Security and Environmental Analysis, Optical Society of America, (2006).

[4] Yun, S. H., Teamey, G. J., de Boer, J. F., Iftimia, N. and Bouma, B. E. , "High-speed optical frequency-domain imaging," Opt. Express 11, 2953-2963 (2003).

[5] Huber, R., Wojtkowski, M. and Fujimoto, J. G. , "Fourier Domain Mode Locking (FDML): A new laser operating regime and applications for optical coherence tomography," Opt. Express 14, 3225-3237 (2006).

[6] Liu, G. Y., Mariampillai, A., Standish, B. A., Munce, N. R., Gu, X. and Vitkin, I. A. , "High power wavelength linearly swept mode locked fiber laser for OCT imaging," Opt. Express 16, 14095-14105 (2008).

[7] Huber, R., Adler, D. C., Srinivasan, V. J. and Fujimoto, J. G. , "Fourier domain mode locking at $1050 \mathrm{~nm}$ for ultrahigh-speed optical coherence tomography of the human retina at 236,000 axial scans per second," Opt. Lett. 32, 20492051 (2007).

[8] Klein, T., Wieser, W., Biedermann, B. R., Eigenwillig, C. M., Palte, G. and Huber, R., "Raman-pumped Fourierdomain mode-locked laser: analysis of operation and application for optical coherence tomography," Opt. Lett. 33, 28152817 (2008).

[9] Jeon, M. Y., Zhang, J., Wang, Q. and Chen, Z. , "High-speed and wide bandwidth Fourier domain mode-locked wavelength swept laser with multiple SOAs," Opt. Express 16, 2547-2554 (2008).

[10] Marhic, M. E., [Fiber Optical Parametric Amplifiers, Oscillators and Related Devices], Cambridge University Press, (2007).

[11] Korotky, S. K., Hansen, P. B., Eskildsen, L. and Veselka, J. J. , "Efficient phase modulation scheme for suppressing stimulated Brillouin scattering," Tech. Dig. International Conf. Integrated Optics and Optical Fiber Communications, Hong Kong, 110-111 (1995).

[12] Marhic, M. E., Wong, K. K. Y. and Kazovsky, L. G. , "Fiber optical parametric amplifiers with linearly or circularly polarized waves," J. Opt. Soc. Am. B 20, 2425-2433 (2003).

[13] Marhic, M. E., Wong, K. K. Y. and Kazovsky, L. G., "Wide-Band Tuning of the Gain Spectra of One-Pump Fiber Optical Parametric Amplifiers," IEEE JSTQE 10, 1133-1141 (2004). 